\title{
Telemedicine for diabetic retinopathy screening using an ultra-widefield fundus camera
}

\author{
This article was published in the following Dove Press journal: \\ Clinical Ophthalmology \\ 14 August 2017 \\ Number of times this article has been viewed
}

\author{
Nazimul Hussain' \\ Maryam Edraki \\ Rima Tahhan ${ }^{2}$ \\ Nishanth Sanalkumar ${ }^{2}$ \\ Sami Kenz ${ }^{2}$ \\ Nagwa Khalil Akasha ${ }^{2}$ \\ Brian Mtemererwa ${ }^{2}$ \\ Nahed Mohammed ${ }^{2}$ \\ 'Department of Ophthalmology, \\ Al Zahra Hospital, Sharjah, United \\ Arab Emirates; ${ }^{2}$ Department of \\ Endocrinology, Al Zahra Hospital, \\ Sharjah, United Arab Emirates
}

\begin{abstract}
Objective: Telemedicine reporting of diabetic retinopathy (DR) screening using ultra-widefield (UWF) fundus camera.

Materials and methods: Cross-sectional study of diabetic patients who visited the endocrinology department of a private multi-specialty hospital in United Arab Emirates between April 2015 and January 2017 who underwent UWF fundus imaging. Fundus pictures are then accessed at the Retina Clinic in the Department of Ophthalmology. Primary outcome measure was incidence of any form of DR detected. The secondary outcome measure was failure to take good image and inability to grade.

Results: A total of 1,024 diabetic individuals were screened for DR from April 2015 to January 2017 in the department of Endocrinology. Rate of DR was 9.27\%; 165 eyes of 95 individuals were diagnosed to have some form of DR. Mild non-proliferative DR (NPDR) was seen in 114 of 165 eyes (69.09\%), moderate NPDR in 32 eyes (19.39\%), severe NPDR in six eyes (3.64\%), and proliferative DR (PDR) in 13 eyes (7.88\%). The secondary outcome measure of poor image acquisition was seen in one individual who had an image acquired in one eye that could not be graded due to bad picture quality.

Conclusions: The present study has shown the effectiveness of DR screening using UWF fundus camera. It has shown the effectiveness of trained nursing personnel taking fundus images. This model can be replicated in any private multi-specialty hospital and reduce the burden of DR screening in the retina clinic and enhance early detection of treatable DR.
\end{abstract}

Keywords: telemedicine, ultra-widefield camera, diabetic retinopathy screening

\section{Introduction}

Diabetes mellitus has become a global epidemic. An estimated 3.4 million people died in 2004 as a consequence of diabetes. ${ }^{1,2}$ World Health Organization (WHO) projects that diabetes will be the seventh leading cause of death by 2030 and this would increase disproportionately more in developing countries. ${ }^{2}$ Hence, the impact on the health care system is an important public health issue. Diabetes mellitus is a leading cause of blindness among working age adults and is estimated to be the cause of blindness in $4.8 \%$ of the 37 million people who are blind throughout the world. ${ }^{3}$

Diabetic retinopathy (DR) represents a wide spectrum of microvascular changes in the retina due to high blood sugar levels. It is often asymptomatic until the consequences of long-term microvascular changes affect the onset of visual loss in the form of macular edema and vitreous hemorrhage. For detection of early DR changes by an endocrinologist, or any physician treating diabetes, it has long been the method to refer to an ophthalmologist or retinologist for screening. Over the years, a screening tool using both digital non-mydriatic fundus imaging and conventional mydriatic fundus camera has been used, and has proven to be beneficial. ${ }^{4-7}$
Correspondence: Nazimul Hussain Department of Ophthalmology, Al Zahra Hospital, PO Box 3499, Sharjah, United Arab Emirates

Tel +97l 65168888

Fax +97I 65637269

Email nazimul.hussain@gmail.com hY $\mathrm{NC}$ and incorporate the Creative Commons Attribution - Non Commercial (unported, v3.0) License (http://creativecommons.org/licenses/by-nc/3.0/). By accessing the work you
hereby accept the Terms. Non-commercial uses of the work are permitted without any further permission from Dove Medical Press Limited, provided the work is properly attributed. For permission for commercial use of this work, please see paragraphs 4.2 and 5 of our Terms (https://www.dovepress.com/terms.php). 
The trends in increasing use of technology worldwide, such as mobile broadband, social media, and increasing e-commerce have allowed health care communities to incorporate information technology in eye care. Telemedicine has evolved from being a research tool to a clinical service over the recent years. ${ }^{8}$ It has helped in screening and detection of diseases allowing prompt treatment thus avoiding severe visual loss.

Incorporating such services between departments in a tertiary care hospital is a big challenge. Screening of all the patients reporting to a retina clinic is also a burden and affects the already waiting, deserving patients with retinal diseases. In this study model, we report telemedicine reporting of DR screening using ultra-widefield (UWF) fundus camera in a private multi-specialty hospital in United Arab Emirates.

\section{Materials and methods}

This is a cross-sectional study of all diabetic patients reporting to the endocrinology department of a private multi-specialty hospital in United Arab Emirates between April 2015 and January 2017. After the patient consults the endocrinologists, the patient is referred to the fundus photography room where trained nursing personnel take the fundus picture using a UWF fundus camera (Optos 200Tx, UWF'M; Optos plc, Dunfermline, UK). The fundus pictures are then accessed at the Retina Clinic in the Department of Ophthalmology of the same hospital. The network connects the UWF fundus camera at endocrinology department to the UWF fundus camera at the retina clinic. The software enables the trained retina specialist to access the image, evaluate and comment. Any comments, along with the fundus picture with patient identification number in PDF format, are placed in the report folder which is accessed by the trained nurse in the endocrinology department. The report is then given to the patient. Based on the comment, the patient is either referred to the retina specialist immediately, appointment is given or review fundus screening as mentioned in the report (Figure 1). Comments include the grading of DR and advice. If any changes were seen in the macula associated with retinal thickening, the report would include diagnosis of clinically significant macular edema. Such patients would visit the retina clinic and be assessed with optical coherence tomography and fundus fluorescein angiography for management.

The fundus imaging was performed without pupil dilation. Several images are taken and the best image was chosen for evaluation. The fundus camera obtains widefield images of $180^{\circ}$ to $200^{\circ}$ through an undilated pupil. A sharp image with high contrast is obtained. The software of Optomap (Optos plc) allows basic image manipulation to evaluate the image in details, using tools such as zooming, red free, infrared, brightness, and contrast. The grader may decide not to grade if the image quality is poor and instead advise to repeat the procedure for proper evaluation.

Grading was based on International Clinical Diabetic Retinopathy Disease Severity Scale ${ }^{9}$ and recommendations are based on the International Clinical Diabetic Retinopathy Disease Severity Scale and the American Academy of Ophthalmology preferred practice pattern guidelines ${ }^{18}$ (Table 1). The primary outcome measure was the incidence of any form of DR detected in the tertiary care multi-specialty private hospital. The secondary outcome measure was failure to take good image and an inability to grade.

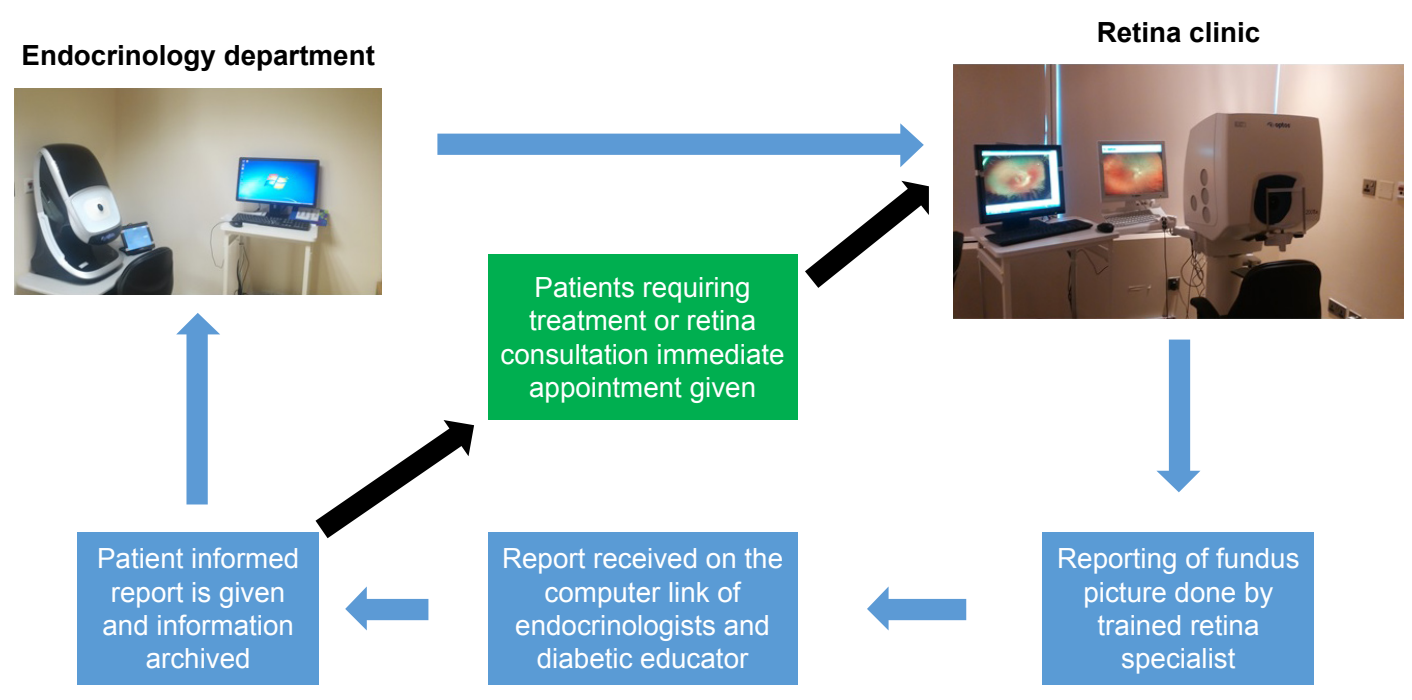

Figure I The protocol and flow chart of screening process. 
Table I Diabetic Retinopathy Disease Severity Scale and International Clinical Diabetic Retinopathy Disease Severity Scale ${ }^{18}$

\begin{tabular}{ll}
\hline Disease severity level & Findings observable upon dilated ophthalmoscopy \\
\hline $\begin{array}{l}\text { No apparent retinopathy } \\
\text { Mild NPDR (see Glossary) }\end{array}$ & No abnormalities \\
$\begin{array}{l}\text { Moderate NPDR (see Glossary) } \\
\text { Severe NPDR }\end{array}$ & More than just microaneurysms but less than severe NPDR \\
US definition & Any of the following (4-2-I rule) and no signs of proliferative retinopathy: \\
& - Severe intraretinal hemorrhages and microaneurysms in each of four quadrants \\
& - Definite venous beading in two or more quadrants \\
International definition & - Moderate IRMA in one or more quadrants \\
& Any of the following and no signs of proliferative retinopathy: \\
& - More than 20 intraretinal hemorrhages in each of four quadrants \\
PDR & - Definite venous beading in two or more quadrants \\
& - Prominent IRMA in one or more quadrants \\
& One or both of the following: \\
& - Neovascularization \\
& - Vitreous/preretinal hemorrhage \\
\hline
\end{tabular}

Notes: Any patient with two or more of the characteristics of severe NPDR is considered to have very severe NPDR. PDR may be classified as high-risk and non-highrisk. American Academy of Ophthalmology Retina/Vitreous Panel. Preferred Practice Pattern Guidelines. Diabetic Retinopathy. San Francisco, CA: American Academy of Ophthalmology; 20I4. Available from: www.aao.org/ppp. ${ }^{18}$

Abbreviations: DR, diabetic retinopathy; NPDR, non-proliferative diabetic retinopathy; PDR, proliferative diabetic retinopathy; IRMA, intraretinal microvascular abnormality.

Approval of the Al Zahra Hospital Ethical committee was obtained for reviewing of data, preparation of manuscript, and permission to publish. Informed consent for examination of patient and inclusion in the study was obtained from every patient.

\section{Results}

Consecutive diabetic individuals $(1,024$ [2,048 eyes]) screened for DR from April 2015 to January 2017 in the department of Endocrinology were included in the study evaluation.

Ninety-five of the 1,024 patients screened were diagnosed to have some form of DR. The incidence rate of DR in this study was $9.27 \%$. The age ranged from 24-77 years with mean age of $50.62 \pm 10.13$ years. The mean duration of diabetes was $12.77 \pm 6.57$ years. Eventually 165 eyes of 95 diabetic individuals were diagnosed to have some form of DR. The distribution of severity grade of DR in 165 eyes were: mild non-proliferative DR (NPDR) in 114 of 165 eyes (69.09\%); moderate NPDR in 32 eyes (19.39\%); severe NPDR in six eyes (3.64\%); and proliferative DR (PDR) either with or without earlier scatter laser in 13 eyes (7.88\%) (Figure 2).

The distribution of severity of DR with similar grading in both eyes or combination of severity scale is shown in Figure 3. Forty-five of the 95 individuals (48.42\%) had mild NPDR in both eyes, $13(13.68 \%)$ had moderate NPDR in both eyes, three (3.16\%) had severe NPDR in both eyes, 7 (7.37\%) had PDR in both eyes, and $26(27.37 \%)$ had a combination of different severity scale in either eye.
The secondary outcome measure of failure to take good image showed that one individual had image acquired in one eye which could not be graded due to bad picture quality. This means that image acquisition with UWF camera by a trained nursing personnel is feasible and this model can be replicated in any private multi-specialty hospital.

\section{Discussion}

Teleophthalmology is practiced all over the world to improve access to care and, at many times to facilitate the prioritization in specialty care. Studies have also shown patient's satisfaction with telemedicine mode of health care delivery. ${ }^{8,10}$

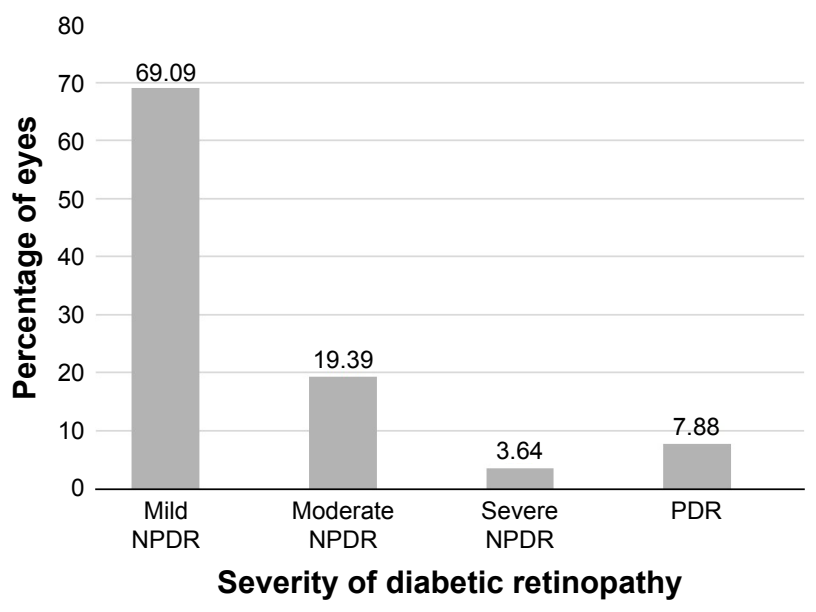

Figure 2 Distribution of percentage of eyes $(n=165)$ using diabetic retinopathy severity scale.

Abbreviations: NPDR, non-proliferative diabetic retinopathy; PDR, proliferative diabetic retinopathy. 


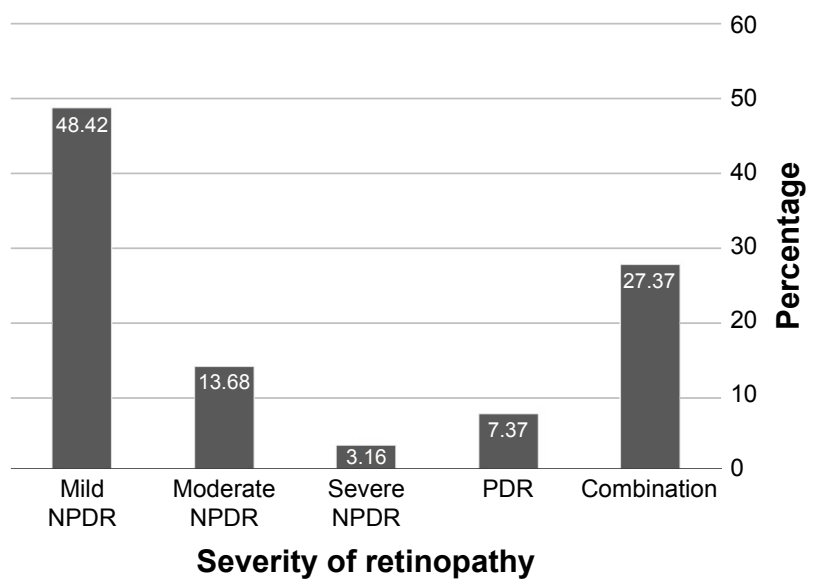

Figure 3 The distribution of severity of diabetic retinopathy in 95 individuals with similar grading in both eyes or combination of severity scale.

Abbreviations: NPDR, non-proliferative diabetic retinopathy; PDR, proliferative diabetic retinopathy.

It has been shown that teleophthalmology produces same desired clinical outcome as the traditional system. ${ }^{8}$

DR screening forms an important part in the prevention and management of microvascular complications of diabetes mellitus. Quellec et $\mathrm{al}^{7}$ have shown the suitability of use of low cost, handheld, non-mydriatic retinography for DR diagnosis compared to the Nidek-AFC 330 non-mydriatic fundus camera. The study showed that during image acquisitions, patients had difficulty locating the fixation target inside the handheld device. This impacted the duration of image acquisition. Thus, $20.4 \%$ of eyes were inadequate for interpretation as compared to Nidek-AFC 330 (9.3\%). However, graders did not find any difference in grading of DR severity. The disadvantage of such cameras is that it gives only a $50^{\circ}$ field and hence, multiple images need to be taken to cover the seven field areas.

Malerbi et a ${ }^{5}$ reported the comparison between binocular indirect ophthalmoscopy (BIO) and digital retinography for DR screening in a multicenter Brazilian type 1 diabetes study. A substantial agreement (kappa 0.67-0.74) was found between BIO and mydriatic retinographies for DR severity but only moderate agreement (kappa 0.58 ) was observed between BIO and non-mydriatic retinography. The main drawback that prevented DR severity detection was poor mydriasis in non-mydriatic retinography.

Our study has shown using non-mydriatic UWF fundus camera, screening 1,024 patients an incidence rate of $9.27 \%$ of DR; 95 patients were detected to have some form DR. The majority of the eyes $(69.09 \%)$ had mild NPDR (Figures 2 and 3). Only 3.64\% of eyes had severe NPDR and $7.88 \%$ had PDR. So less than $12 \%$ of eyes needed further investigations and management. This means that these eyes had the advantage of immediate management to prevent further progressive retinopathy. The advantage is clearly highlighted that these patients were either ignorant about diabetes affecting the eye and probably their vision was not affected so to require eye examination. Furthermore, only one individual had image acquired in one eye which could not be graded due to bad picture quality. This indicates that image acquisition with UWF camera by trained nursing personnel is feasible and this model can be replicated in any private multi-specialty hospital.

Kernt et $\mathrm{al}^{11}$ has shown the advantage of assessment of DR using non-mydriatic UWF scanning laser ophthalmoscopy (Optomap) compared with ETDRS 7-field stereo photography. Agreement between readers were higher for Optomap then 7-field photography in assessing clinically significant macular edema (CSME) with exact agreement of $91 \%$ vs $87.5 \%$. Kappa was 0.89 ( $\mathrm{SE}=0.03$ ) for Optomap and $0.84(\mathrm{SE}=0.04)$ for 7-field photography. For DR grading levels, a similar level of agreement was found.

Authors ${ }^{11-14}$ have enumerated that limitations of mydriatic 7-field stereo photography is time consuming and highly dependent on the photographer's experience and compliance of the patient. So, non-mydriatic digital fundus imaging has the advantage of patient comfort, no need for dilation, and facilitates remote diagnostic image generation and interpretation. These factors improve patient compliance. UWF Optomap imaging allows non-mydriatic imaging of the posterior pole as well as imaging extending over the equator $\left(180^{\circ}-200^{\circ}\right)$. Besides, it also allows manipulation with red free and infrared image and selectively visualizes the deeper and superficial neurosensory retina. Theoretically, it is more advantageous than 7-field photography avoiding multiple image acquisition.

Several prospective studies have shown very good correlation between different imaging modalities with UWF imaging. ${ }^{15}$ Silva et al ${ }^{16}$ have reported UWF imaging increased the identification of DR (38.4\% vs $33.8 \%, P=0.0053)$ and vision threatening DR (14.5\% vs $11.9 \%)$ compared to non-mydriatic fundus photography. Moreover, peripheral retinal lesions could be detected located outside the ETDRS 7-standard field in more severe assessment of the level of DR in $9 \%$ of patients and neovascularization elsewhere (NVE) which were not seen on ETDRS 7-field photography. It has also been shown that the acquisition time using UWF imaging is significantly shorter compared to ETDRS 7-standard field photography. ${ }^{16,17}$

So, telemedicine based DR screening has grown steadily for last few decades and has become well established. ${ }^{1}$ 
Advancement in technology over the years has also improved the efficiency and effectiveness of digital screening.

The present study has shown the effectiveness of DR screening using UWF fundus camera detecting $9.27 \%$ of patients with DR. It has also shown the effectiveness of trained nursing personnel taking fundus pictures. The available studies reported and the present review of telemedicine and evaluation of fundus pictures highlights the advantage of UWF imaging. First, UWF imaging has a fast learning curve and it is quick to take the picture. Second, widefield view is greater than the 7-field photography enabling more DR lesions to be detected (Figures 4-7). And third, images can be manipulated in terms of red free, infra-red and image zoom. Hence, the present telemedicine model has demonstrated that in a multi-specialty private hospital, DR screening can be effective using UWF fundus camera. This will reduce the screening burden in the retina clinic and allow the retina specialist to attend to more deserving patients with treatable retinal disease. This also improves the compliance of

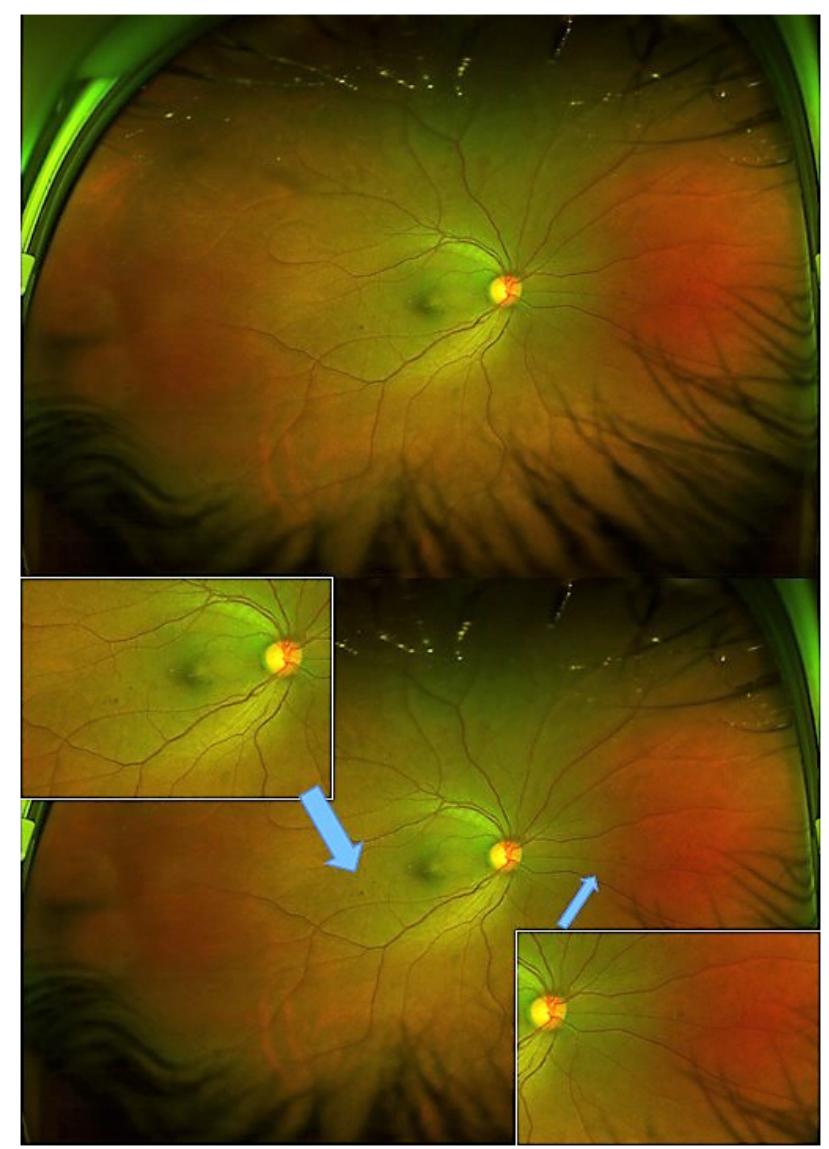

Figure 4 Shows the wide field of view of fundus with few dot retinal hemorrhages suggestive of mild NPDR (top). Bottom image shows the inset magnified views of the lesion areas (blue arrows), an advantage of this technology in manipulating the image to detect lesions more effectively.

Abbreviation: NPDR, non-proliferative diabetic retinopathy.

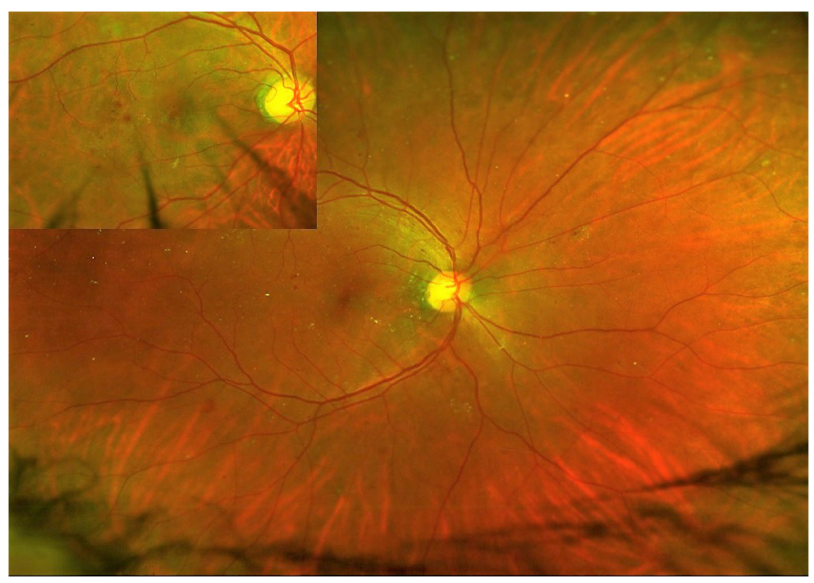

Figure 5 Widefield view of fundus suggestive of moderate NPDR. Inset shows the magnified view of one lesion area.

Abbreviation: NPDR, non-proliferative diabetic retinopathy.

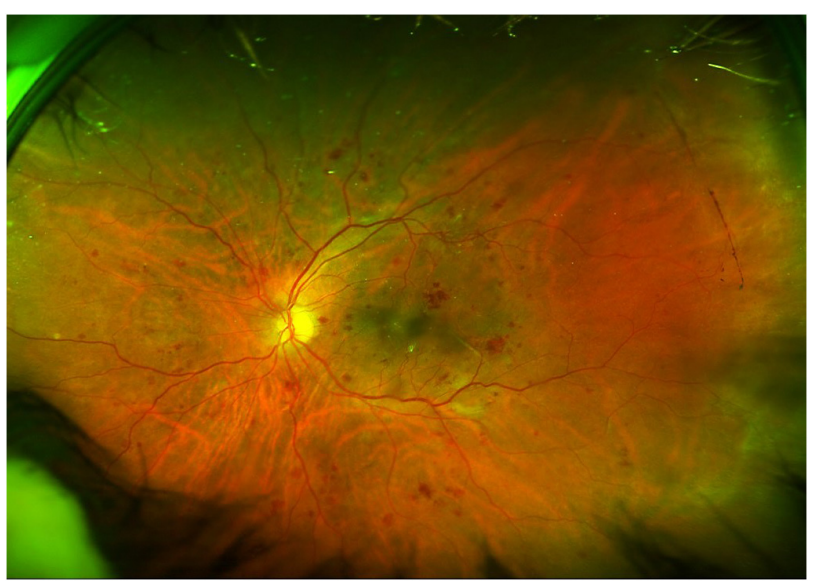

Figure 6 Widefield view of fundus suggestive of severe NPDR. Note the ability to visualize lesions beyond the standard 7-field photograph. Peripherals lesions are effectively seen.

Abbreviation: NPDR, non-proliferative diabetic retinopathy.

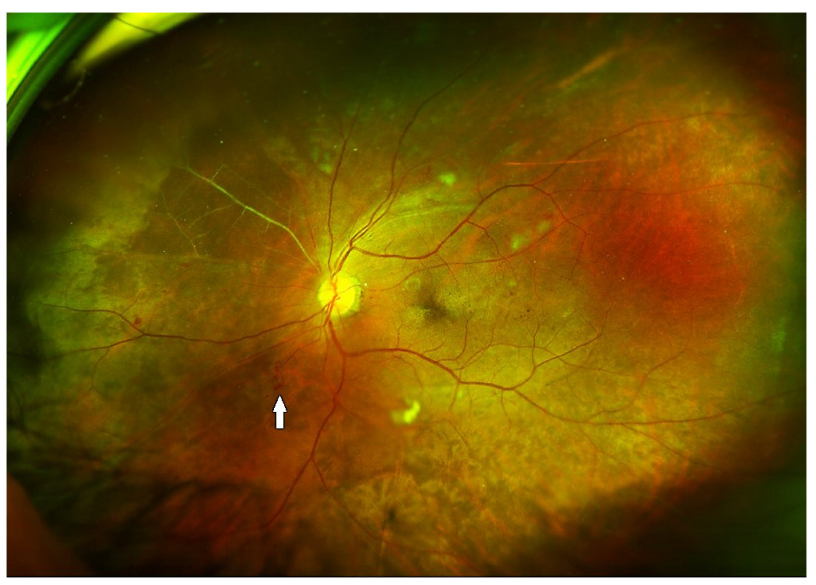

Figure 7 Widefield view of fundus suggestive of PDR. Arrow shows a retinal neovascularization elsewhere, and note the extent of occluded vessels seen in the nasal quadrant.

Abbreviation: PDR, proliferative diabetic retinopathy. 
the patient by completing retina exam when they come for check up in the diabetic clinic and increases the detection of undiagnosed retinopathy in the diabetic who would not otherwise visit a retinologist.

The limitation of the present study is the population screened for DR do not represent the actual population at risk as it was a hospital based screening. The disadvantages of the technology being used, even though promising and effective, is the cost of the fundus camera and time availability of the retinologists to read the images and report. If possible, this technology can be extrapolated to replace the present screening methodology and increase the detection of DR due to its known advantages. Since the technology is easy to learn and user friendly, the learning curve of any non-ophthalmologist is short. Hence, any available nursing personnel in the department could be trained avoiding the need for recruitment. Gross media opacity like cataract or vitreous hemorrhage may hamper fundus imaging but such issues are encountered in any imaging technology. Such patients could be then referred to the specialized clinic for further retina evaluation.

\section{Conclusion}

This model can be replicated in any multi-specialty hospital to reduce the burden of DR screening in retina clinics and enhance early detection of treatable DR. It has shown the effectiveness of DR screening using UWF fundus camera and effectiveness of trained nursing.

\section{Disclosure}

The authors report no conflicts of interest in this work.

\section{References}

1. World Health Organization. Global Health Risks. Mortality and Burden of Disease Attributable to selected Major Risks. World Health Organization, Geneva, Switzerland. 2009.

2. Das T, Raman R, Ramasamy K, Rani PK. Telemedicine in diabetic retinopathy: current status and future directions. Middle East Afr $J$ Ophthalmol. 2015;22(2):174-178.
3. World Health Organization. Prevention of blindness from diabetes mellitus: report of a WHO Consultation. World Health Organization, Geneva, Switzerland. 2005.

4. Taylor RC, Merin LM, Salunga AM, et al. Improving diabetic retinopathy screening ratios using telemedicine-based digital retinal imaging technology. The Vine Hill Study. Diabetes Care. 2007;30:574-578.

5. Malerbi FK, Morales PH, Farah ME, et al. Comparison between binocular indirect ophthalmoscopy and digital retinography for diabetic retinopathy screening: the Brazilian Type I Diabetes Study. Diabetol Metab Syndr. 2015;7:116.

6. Zimmer-Galler IE, Kimura AE, Gupta S. Diabetic Retinopathy screening and use of telemedicine. Curr Opin Ophthalmol. 2015;26(3): 167-172.

7. Quellac G, Bazin L, Cazuguel G, Delafoy I, Cochener B, Lamaed M. Suitability of a low cost, hand held, non-mydriatic retinograph for diabetic retinopathy diagnosis. Trans Vis Sci Tech. 2016;5(2):16.

8. Sreelatha OK, Ramesh SVS. Teleophthalmology: improving patient outcomes? Clin Ophthalmol. 2016;10:285-295.

9. Wilkinson CP, Ferris FL, Klein RE, et al. Proposed international clinical diabetic retinopathy and diabetic macular edema disease severity scales. Ophthalmology. 2003;110:1677-1682.

10. Mair F, Whitten P. Systematic review of studies of patient satisfaction with telemedicine. BMJ. 2000;320(7248):1517-1520.

11. Kernt M, Hadi I, Pinter F, et al. Assessment of diabetic retinopathy using non-mydriatic ultra wide field scanning laser ophthalmoscopy (Optomap) compared with ETDRS 7-Fields stereo photography. Diabetes Care. 2012;35:2459-2463.

12. Kernt M, Pinter F, Hadi L, et al. Diabetic retinopathy: Comparison of diagnostic features of Ultra widefield scanning laser ophthalmoscopy optomap with ETDRS 7 Field fundus photography. Ophthalmologe. 2011;108(2):117-123.

13. Danis RP, Hubbard LD. Imaging of diabetic retinopathy and diabetic macular edema. Curr Diab Rep. 2011;11:236-243.

14. Lopez-Bastida J, Cabrera-Lopez F, Serrano-Anguilar P. Sensitivity and specificity of digital retinal imaging for screening diabetic retinopathy. Diabet Med. 2007;24:403-407.

15. Tan CS, Chew MC, Lim LW, Sadda SR. Advances in retinal imaging for diabetic retinopathy and diabetic macular edema. Indian J Ophthalmol. 2016;64:76-83.

16. Silva PS, Cavallerano JD, Tolls D, et al. Potential efficiency benefits of non-mydriatic ultrawide field retinal imaging in an ocular telehealth diabetic retinopathy program. Diabetes Care. 2014;37:50-55.

17. Silva PS, Cavallerano JD, Sun JK, Noble J, Aiello LM, Aiello LP. Nonmydriatic ultrawide field retinal imaging compared with dilated standard 7-field $35 \mathrm{~mm}$ photography and retinal specialist examination for evaluation of diabetic retinopathy. Am J Ophthalmol. 2012;154:549-559.

18. American Academy of Ophthalmology Retina/Vitreous Panel. Preferred Practice Pattern Guidelines. Diabetic Retinopathy. San Francisco, CA: American Academy of Ophthalmology; 2014. Available from: www. aao.org/ppp. Accessed June 19, 2017.
Clinical Ophthalmology

\section{Publish your work in this journal}

Clinical Ophthalmology is an international, peer-reviewed journal covering all subspecialties within ophthalmology. Key topics include: Optometry; Visual science; Pharmacology and drug therapy in eye diseases; Basic Sciences; Primary and Secondary eye care; Patient Safety and Quality of Care Improvements. This journal is indexed on Submit your manuscript here: http://www.dovepress.com/clinical-ophthalmology-journal

\section{Dovepress}

PubMed Central and CAS, and is the official journal of The Society of Clinical Ophthalmology (SCO). The manuscript management system is completely online and includes a very quick and fair peer-review system, which is all easy to use. Visit http://www.dovepress.com/ testimonials.php to read real quotes from published authors. 